\title{
THE INFLUENCE OF THE WEIGHT FUNCTION ON THE RESULTS ACCURACY IN STRUCTURAL ANALYSIS BY EFG METHOD
}

\author{
Vasile NĂSTĂSESCU*, Ghiță BÂRSAN**, Silvia MARZAVAN* \\ *"Ferdinand I" Military Technical Academy, Bucharest, Romania \\ **"Nicolae Bălcescu" Land Forces Academy, Sibiu, Romania \\ vasile.nastasescu@mta.ro
}

\begin{abstract}
The use of mesh free methods, including the EFG method, raises a number of user issues. For the best possible result, the user must choose both the weight function and a series of parameters of the method used. This paper presents a part of the research undertaken by the authors to substantiate, on the basis of numerical experiments, some conclusions that will lead the user as quickly as possible to a more accurate result, which can be validated by analytical results and often by experiment. The numerical experiments performed by the authors are based on the use of their own program, made in Matlab, which allowed the use of many weighting functions, in different conditions established by choosing several values of the internodal distance and the size of the support domain. The numerical experiment is performed on a simple structure, widely used in the technical literature under the name Timoshenko beam, for which there are analytical (exact) solutions for both the displacements and stresses. The obtained results are presented in graphical and tabular form, with the highlighting of the errors compared to the exact solution. The final conclusions provide valuable information for practical work with the EFG method, which are also valid for the use of other meshless methods.
\end{abstract}

\section{Keywords: weight function, support domain, EFG method, field nodes, Gauss points}

\section{Introduction}

Due to the complexity of the phenomena, the use of numerical methods is a necessity. Of these methods, FEM is one of the best known, most used and most developed. However, FEM, like all other numerical methods of structure analysis, has some drawbacks, upon which we do not comment on in this paper. But this observation may explain, even if only partially, the concern of specialists to produce and develop new numerical methods. Meshless methods are those that have been imposed in the practice of structure calculus and in general, of continuous medium calculus.

A meshfree methodis a method used to establish a system of algebraic equations for the whole domain of problem, without using a predefined mesh for the domain discretization. Such a method uses a set of scattered nodes, called field nodes, to establish the problem domain and boundaries.

According to the formulation procedure, meshfree methods fall into three categories: meshfree weak form methods (EFG and others) meshfree strong form methods and meshfree weak-strong form methods based on the combination of both weak form and strong form.

In the meshfree weak form methods, the governing partial differential equations with derivative boundary conditions are first transformed to a set of so called weak form 
integral equations using different techniques. The weak forms are then used to derive a set of algebraic equation system by a numerical integration process using sets ofbackground cells, that may be constructed globally or locally, in the problem domain.

The EFG method is a typical meshfree global weak form method. The boundary conditions can be of the Dirichlet, Neumann or Dirichlet and Neumann type; the best results of their using are obtained by the respectively meshfree methods, according to the above classification.

In meshfree methods, regardless of the type of formulation, to approximate the field variables, shape functions or weight functions are used, which must meet certain conditions. The weight function and also its parameters substantially influence the accuracy of the results.

\section{EFG Method Fundamentals}

In the Element Free Galerkin (EFG) method, the moving least square (MLS) method is used for constructing the weight (shape) functions.

This procedure was first time proposed by Lancaster and Salkauskas as an interpolation method. As the evaluation methods are concerned, two methods are used: interpolation methods (FEM) and approximation methods (EFG method). The difference between these methods is illustrated by the Figure 1. In interpolation procedure, the nodal function values are the same with the node values (Figure 1-a).

In the evaluation procedure, the nodal function values are not the same with the nodal values (Figure 1-b).

Many weight functions [3], [9], [10] have been designed, starting from their properties which must be full filled. Without detailing them, the main properties are: positivity, compactness, unity, monotonic decreasing, delta function behavior and convergence property.

Referring to the weight functions, the literature presents many such functions.

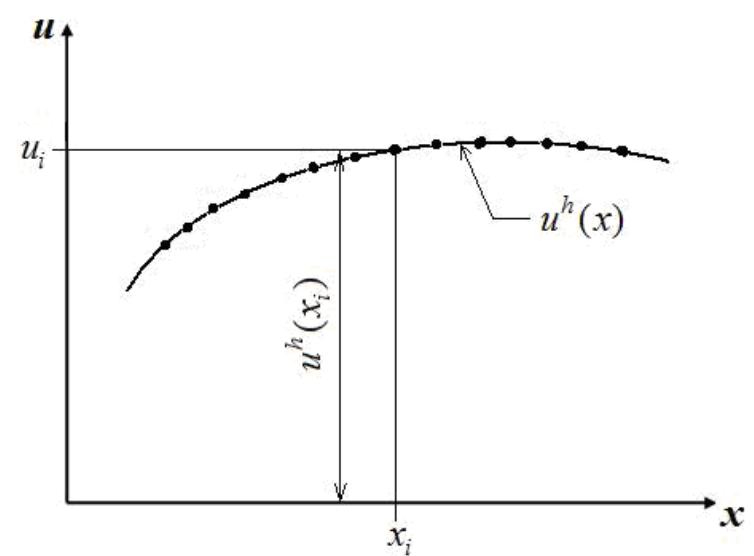

a) interpolation

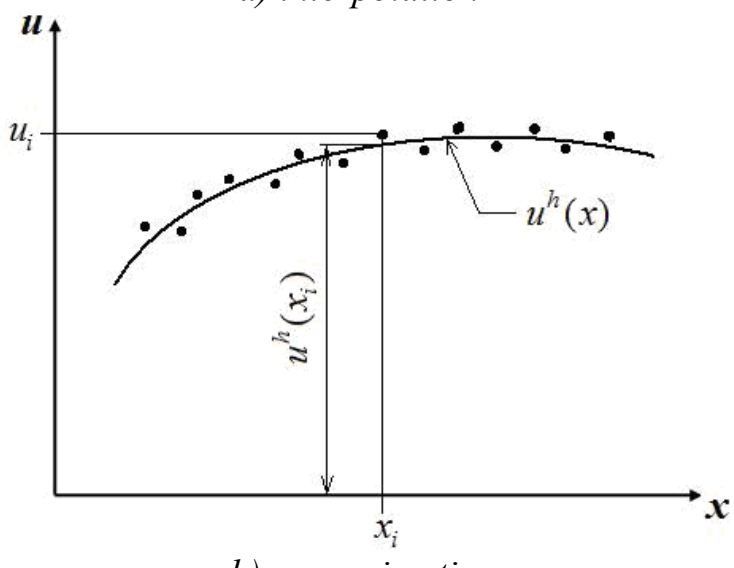

b) approximation

Figure 1: Methods of evaluation in numerical methods

As the EFG method is concerned, the most used weight functions are:

- cubic spline weight function,

$$
w(r)=\left\{\begin{array}{lr}
\frac{2}{3}-4 r^{2}+4 r^{3} & r \leq 0.5 \\
\frac{4}{3}-4 r^{2}-\frac{4}{3} r^{3} & 0.5<r \leq 1 \\
0 & r>1
\end{array}\right.
$$

- cubic weight function,

$$
w(r)=\left\{\begin{array}{lr}
1-3 r^{2}+2 r^{3} & 0 \leq r \leq 1 \\
0 & r>1
\end{array}\right.
$$

- quartic weight function,

$$
w(r)= \begin{cases}1-6 r^{2}+8 r^{3}-3 r^{4} & r \leq 1 \\ 0 & r>1\end{cases}
$$

- new quartic weight function, 


$$
w(r)= \begin{cases}\frac{2}{3}-\frac{9}{2} r^{2}+\frac{19}{3} r^{3}-\frac{5}{2} r^{4} & r \leq 1 \\ 0 & r>1\end{cases}
$$

- Johnson quadratic weight function,

$$
w(r)=\frac{3}{4}-\frac{3}{4} r+\frac{3}{16} r^{2} \quad 0 \leq r \leq 2
$$

This weight function is recommended for solving of the impact problems.

In all weight functions presented here, the variable $r$ is a vector defining the relative distance between a point of interest and other points around it, by the relation (15),

$$
(r)_{I}=\frac{\left\|x-x_{I}\right\|}{d_{m I}}
$$

where $x_{I}$ is the coordinate of the point of interest, $x$ is the coordinate of a neighbouring point; $d_{m I}$ is the size of the influence domain of node $I$ (Figure 2).

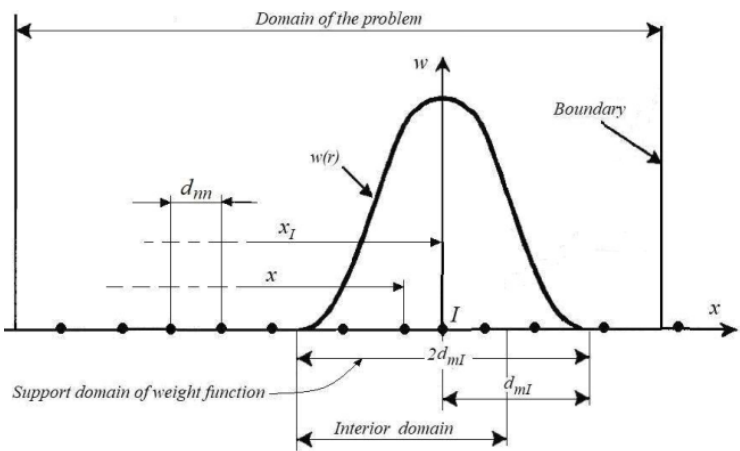

Figure 2: Weight function for $1 D$ problem

Graphical representation of these weight functions is presented in the Figure 3.

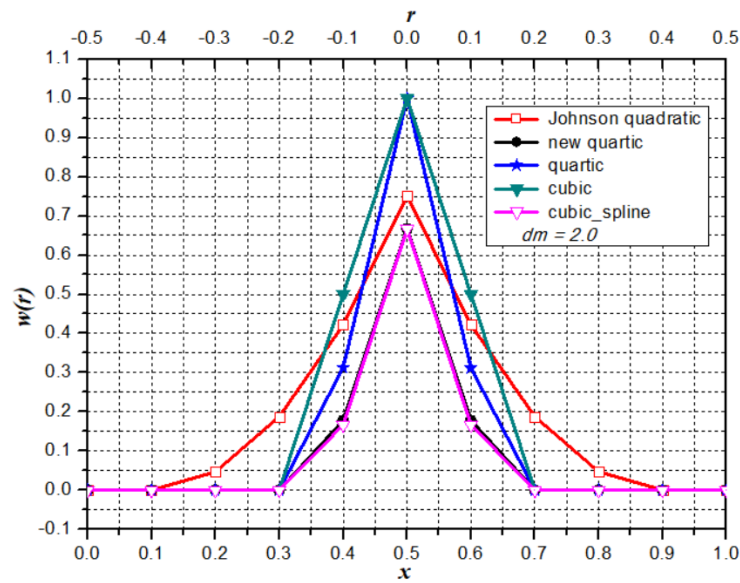

Figure 3: Weight functions in EFG method
For 2D-problems, the weight function is a surface like in the Figure 4. The support domain can have a circular or quadrilateral shape (square or rectangle).

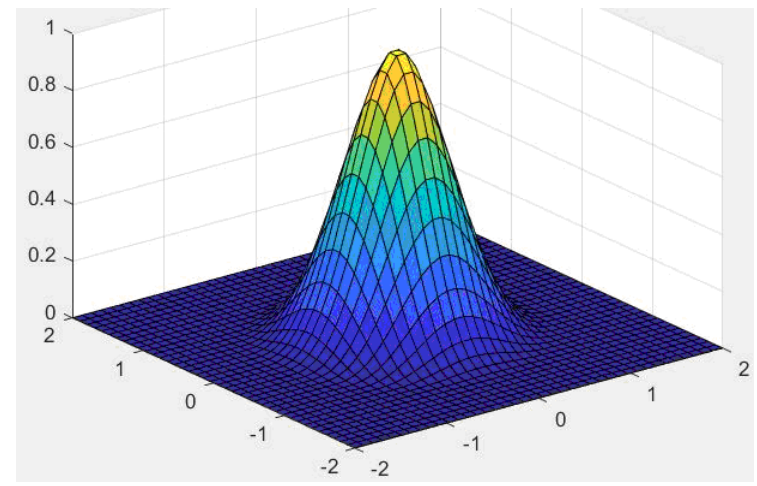

Figure 4: Weight function for a $2 D$ problem

Any field parameter, in a field point of coordinates $x_{i}$, is expressed by the relation,

$$
u\left(x_{i}\right)=\sum_{i=1}^{n} \Phi_{i} u_{i}
$$

where $\Phi_{i}$ is the weight function in the $i$ point. The nodal discrete equations are obtained using the constrained Galerkin weak form and the Lagrange multiplier for imposing the essential boundary conditions on the problem domain $\Omega$.

Following are the governing matrices for boundary condition, stiffness, force and displacement respectively.

$$
\begin{aligned}
& K_{I J}=\int_{\Omega} B_{I}^{T} D B_{J} \mathrm{~d} \Omega \\
& G_{I K}=-\int_{\Gamma_{u}} \Phi_{i} N_{k} \mathrm{~d} \Gamma \\
& f_{I}=\int_{\Gamma_{t}} \Phi_{i} t \mathrm{~d} \Gamma+\int_{\Omega} \Phi_{i} b \mathrm{~d} \Omega \\
& q_{K}=-\int_{\Gamma_{u}} N_{K} u \mathrm{~d} \Gamma
\end{aligned}
$$

In the above relations the following notations were used, for the case of 2D stress and displacement state:

$$
B_{I}=\left[\begin{array}{cc}
\Phi_{I, x} & 0 \\
0 & \Phi_{I, y} \\
\Phi_{I, y} & \Phi_{I, x}
\end{array}\right]
$$




$$
\begin{aligned}
& N_{K}=\left[\begin{array}{cc}
N_{K} & 0 \\
0 & N_{K}
\end{array}\right] \\
& D=\frac{E}{1-v^{2}}\left[\begin{array}{ccc}
1 & v & 0 \\
v & 1 & 0 \\
0 & 0 & \frac{1-v}{2}
\end{array}\right]
\end{aligned}
$$

$E$ and $v$ are Young's modulus and Poison's ratio respectively. The above discrete nodal equations are assembled into global matrix, in a well known form,

$$
[K] \cdot\{u\}=\{F\}
$$

which in EFG method is written:

$$
\left[\begin{array}{cc}
K & G \\
G^{T} & 0
\end{array}\right] \cdot\left[\begin{array}{l}
u \\
\lambda
\end{array}\right]=\left[\begin{array}{l}
f \\
q
\end{array}\right]
$$

\section{Organizing the numerical experiment}

The numerical experiment was performed using a problem well known in the scientific literature, namely Timoshenko beam (Figure 5).

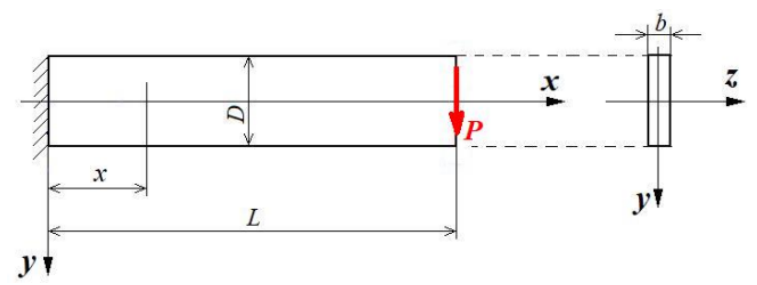

Figure 5: Analytical model of Timoshenko beam

The problem data are: $L=0.48 \mathrm{~m}$, $D=0.12 \mathrm{~m}, \quad b=0.10 \mathrm{~m}, \quad E=3 \cdot 10^{7} \mathrm{~Pa}$, $v=0.30$ and $I_{z} \equiv I=1.44 \cdot 10^{-5} \mathrm{~m}^{4}$.

By numerical experiment the maximum displacement $u_{y}$ and the maximum stress $\sigma_{x}$ where investigated, using different weight functions, different internodal distances and support domain sizes.

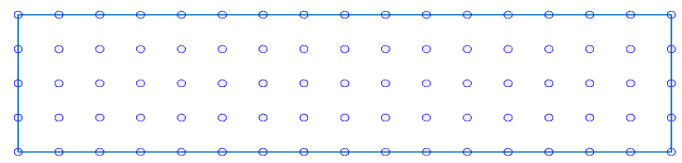

a) internodal distance $d_{n n}=0.03 \mathrm{~m}$

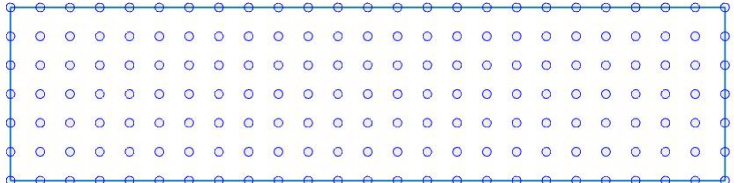

b) internodal distance $d_{n n}=0.02 \mathrm{~m}$

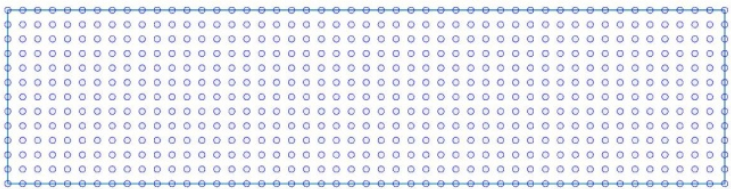

c) internodal distance $d_{n n}=0.01 \mathrm{~m}$

Figure 6: The distribution of the field nodes

Most of the time, the influence domain coincides with the support domain (Figure 2), like in this example. Also, in this example, the field nodes were uniformly distributed in three cases, like in the Figure 6.

The using of the EFG method was done through a program made in Matlab.

For the integration of the equations were used the integration cells shown in Figure 7 , in three variants according to the adopted number of field nodes.

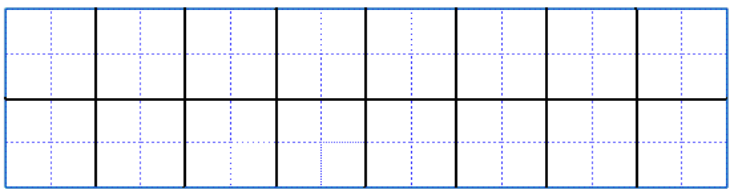

a) quadrature cells for $d_{n n}=0.03 \mathrm{~m}$

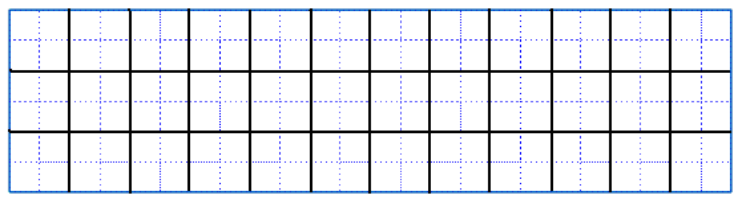

b) quadrature cells for $d_{n n}=0.02 \mathrm{~m}$

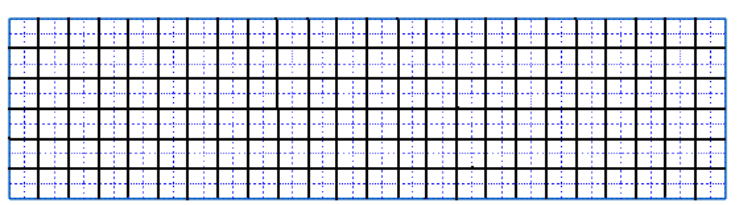

c) quadrature cells for $d_{n n}=0.01 \mathrm{~m}$

Figure 7: Quadrature cells

The sizing and numbering of the integration cells for those three cases of the internodal distance $d_{n n}$ are presented in Figures8...10.In each variant of the field 
node number (Figure 7), each cell contains 16 Gaussian integration points.

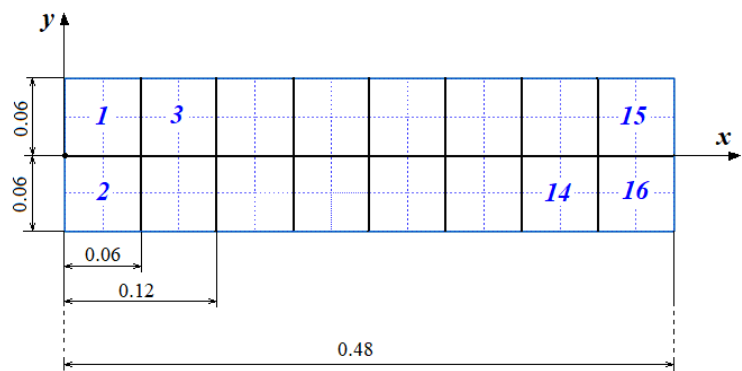

Figure 8: Quadrature cells for $d_{n n}=0.03 \mathrm{~m}$

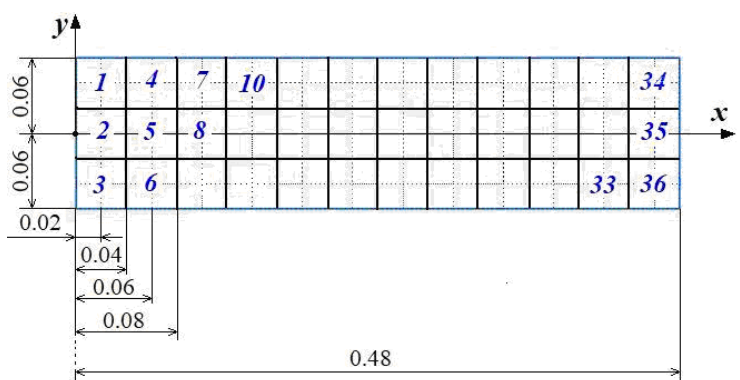

Figure 9: Quadrature cells for $d_{n n}=0.02 \mathrm{~m}$

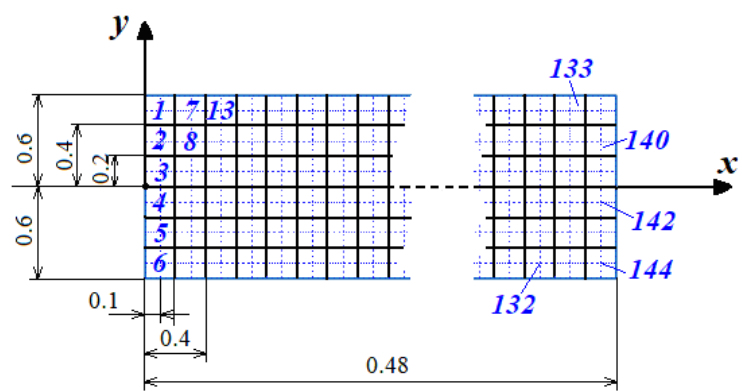

Figure 10 Quadrature cells for $d_{n n}=0.01 \mathrm{~m}$

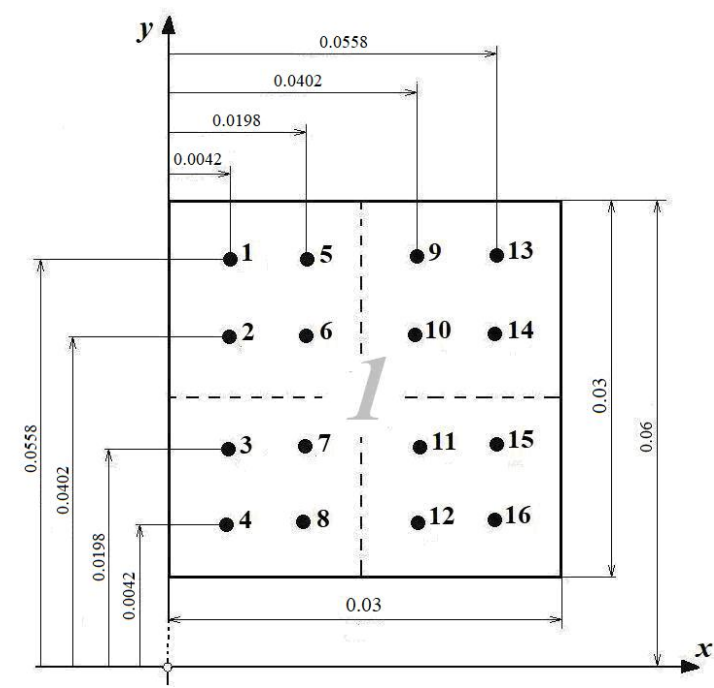

Figure 11. Integration points in the cell no. 1 for an internodal distance $d_{n n}=0.03 \mathrm{~m}$.
The numbering of Gaussian points in a cell is the same in all cases, but their coordinates vary depending on the internodal distance $d_{n n}$. For cell 1 of Figure 8 , the Gaussian integration points are shown in Figure 11.

The maximum transverse displacement of the beam node is calculated by the relation:

$u_{y_{\max }} \frac{P}{6 E I}\left[(4+5 v) \frac{D^{2} L}{4}+2 L^{3}\right]$

Analytical solution is provided by the formulas of Timoshenko's Theory of Elasticity [8], [12].

The analytical calculus of the stress $\sigma_{x}$ was performed in the Gauss point, for a direct comparison with the values of the numerical analysis. The relation is,

$\sigma_{x}=-\frac{P(L-x) y}{I}$

where $x$ and $y$ are the coordinates of the respective Gaussian point. Each time, the gauss point (its coordinates) was identified where the stress $\sigma_{x}$ has the maximum value.

\section{The results of the performed research}

The results of the numerical research, representing a large volume of data, were organized tabularly and graphically processed, as presented below.

For each of the versions of the internodal distance considered, for each weight function analyzed, the results were organized in tables, as in Table 1.

There are three such tables (one for each internodal distance considered: $0.01 ; 0.02$ and $0.03 \mathrm{~m}$ ), but only one is presented here, for space saving.

In this paper, only a part of our results are presented. Many others weight functions were studied and the range of $d_{m}$ parameter has been extended to value 4 .

This paper contains the most important results of our research for the practice of the EFG method using. 
Table 1 The results of numerical experiment, for $d_{n n}=0.03 \mathrm{~m}$

\begin{tabular}{|c|c|c|c|c|c|c|}
\hline \multirow{2}{*}{\multicolumn{2}{|c|}{$\begin{array}{l}\text { Analitical } \\
\text { values }\end{array}$}} & \multicolumn{2}{|c|}{$u_{y_{\max }}^{\text {theoretic }}[m]$} & \multicolumn{2}{|c|}{$\sigma_{x_{\max }}^{\text {theoretic }}[P a]$} & \multirow{3}{*}{$\begin{array}{c}\text { Error } \\
\text { by } \\
\text { Energy } \\
\text { Norm }\end{array}$} \\
\hline & & \multicolumn{2}{|c|}{0.088999} & \multicolumn{2}{|c|}{2000000} & \\
\hline \multicolumn{6}{|c|}{ CUBIC SPLINE Weight Function } & \\
\hline \multicolumn{2}{|c|}{$\begin{array}{l}\text { Numerical } \\
\text { values }\end{array}$} & $u_{y_{\max }}$ & $\begin{array}{l}E r r . \\
{[\%]}\end{array}$ & $\sigma_{x_{\max }}$ & $\begin{array}{l}E r r . \\
{[\%]}\end{array}$ & enorm \\
\hline \multirow{7}{*}{$d_{m}$} & 1.25 & 0.086724 & -2.56 & 1807028 & -1.99 & 3.3227 \\
\hline & 1.50 & 0.087883 & -1.25 & 1772408 & -3.87 & 2.2545 \\
\hline & 1.75 & 0.088594 & -0.45 & 1774146 & -3.77 & 1.2787 \\
\hline & 2.00 & 0.088811 & -0.21 & 1786570 & -3.10 & 0.8394 \\
\hline & 2.50 & 0.089002 & 0.00 & 1784656 & -3.20 & 0.4499 \\
\hline & 3.00 & 0.088878 & -0.14 & 1820493 & -1.26 & 0.2523 \\
\hline & 3.50 & 0.089829 & 0.93 & 1768769 & -4.07 & 0.8334 \\
\hline \multicolumn{2}{|c|}{ Total Err } & & .52 & \multicolumn{2}{|c|}{$\begin{array}{lll}-3.04 & 1\end{array}$} & \\
\hline \multicolumn{7}{|c|}{ Q QUARTIC Weight Function } \\
\hline \multicolumn{2}{|c|}{$\begin{array}{c}\text { Numerical } \\
\text { values }\end{array}$} & $u_{y_{\max }}$ & $\begin{array}{l}E r r . \\
{[\%]}\end{array}$ & $\sigma_{x_{\max }}$ & $\begin{array}{l}\text { Err. } \\
{[\%]}\end{array}$ & enorm \\
\hline \multirow{7}{*}{$d_{m}$} & 1.25 & 0.086999 & -2.25 & 1785122 & -3.18 & 3.1072 \\
\hline & 1.50 & 0.088352 & -0.73 & 1767168 & -4.15 & 1.6957 \\
\hline & 1.75 & 0.088812 & -0.21 & 1783256 & -3.28 & 0.9280 \\
\hline & 2.00 & 0.088787 & -0.24 & 1804007 & -2.15 & 0.6414 \\
\hline & 2.50 & 0.088923 & -0.09 & 1780824 & -3.41 & 0.5157 \\
\hline & 3.00 & 0.088880 & -0.13 & 1819568 & -1.31 & 0.2956 \\
\hline & 3.50 & 0.088990 & -0.01 & 1832741 & -0.60 & 0.2977 \\
\hline \multicolumn{2}{|c|}{ Total Err. } & \multicolumn{2}{|c|}{-0.52} & \multicolumn{2}{|c|}{$-2.581 .0688$} & \\
\hline \multicolumn{7}{|c|}{ NEW QUARTICWeight Function } \\
\hline \multicolumn{2}{|c|}{$\begin{array}{l}\text { Numerical } \\
\text { values }\end{array}$} & $u_{y_{\max }}$ & $\begin{array}{l}E r r . \\
{[\%]}\end{array}$ & $\sigma_{x_{\max }}$ & $\begin{array}{l}\text { Err. } \\
{[\%]}\end{array}$ & enorm \\
\hline \multirow{7}{*}{$d_{m}$} & 1.25 & 0.086989 & -2.26 & 1802023 & -2.26 & 3.1399 \\
\hline & 1.50 & 0.087916 & -1.22 & 1763414 & -4.36 & 2.2404 \\
\hline & 1.75 & 0.088750 & -0.28 & 1777940 & -3.57 & 1.1178 \\
\hline & 2.00 & 0.088781 & -0.25 & 1807628 & -1.96 & 0.8252 \\
\hline & 2.50 & 0.088887 & -0.13 & 1769382 & -4.03 & 0.7155 \\
\hline & 3.00 & 0.088982 & -0.02 & 1825604 & -0.98 & 0.4045 \\
\hline & 3.50 & 0.088980 & -0.02 & 1845243 & 0.08 & 0.4036 \\
\hline \multicolumn{2}{|c|}{ Total Err. } & \multicolumn{2}{|c|}{-0.60} & \multicolumn{2}{|c|}{$\begin{array}{rr}-2.44 & 1 \\
\end{array}$} & \\
\hline \multicolumn{7}{|c|}{ CUBIC Weight Function } \\
\hline \multicolumn{2}{|c|}{$\begin{array}{l}\text { Numerical } \\
\text { values }\end{array}$} & $u_{y_{\max }}$ & $\begin{array}{l}\text { Err. } \\
{[\%]}\end{array}$ & $\sigma_{x_{\max }}$ & $\begin{array}{l}\text { Err. } \\
{[\%]}\end{array}$ & enorm \\
\hline & 1.25 & 0.088375 & -0.70 & 1754580 & -4.84 & 1.7487 \\
\hline & 1.50 & 0.088966 & -0.04 & 1826521 & -0.93 & 1.1216 \\
\hline & 1.75 & 0.088702 & -0.33 & 1891412 & 2.59 & 1.1013 \\
\hline$d_{m}$ & 2.00 & 0.089134 & 0.15 & 1752371 & -4.95 & 2.0778 \\
\hline & 2.50 & 0.088465 & -0.60 & 1820374 & -1.27 & 0.7629 \\
\hline & 3.00 & 0.088783 & -0.24 & 1741121 & -5.57 & 1.3770 \\
\hline & 3.50 & 0.082798 & -6.97 & 1854993 & 0.61 & 0.8707 \\
\hline Tot: & Err. & -1. & $5-2.05$ & 1.2943 & & \\
\hline
\end{tabular}




\begin{tabular}{|c|c|c|c|c|c|c|}
\hline \multicolumn{7}{|c|}{ JOHNSON QUADRATIC Weight Function } \\
\hline \multicolumn{2}{|c|}{$\begin{array}{c}\text { Numerical } \\
\text { values }\end{array}$} & $u_{y_{\max }}$ & $\begin{array}{l}\text { Err. } \\
{[\%]}\end{array}$ & $\sigma_{x_{\max }}$ & $\begin{array}{l}\text { Err. } \\
{[\%]}\end{array}$ & enorm \\
\hline \multirow{7}{*}{$d_{m}$} & 1.25 & 0.090647 & 1.85 & 1706883 & -7.42 & 1.8099 \\
\hline & 1.50 & 0.094593 & 6.29 & 1670982 & -9.37 & 1.6800 \\
\hline & 1.75 & 0.098348 & 10.50 & 1816581 & -1.47 & 2.9376 \\
\hline & 2.00 & 0.101538 & 14.09 & 1949111 & 5.72 & 3.2829 \\
\hline & 2.50 & 0.117185 & 31.67 & 1847183 & 0.19 & 5.5458 \\
\hline & 3.00 & 0.121734 & 36.78 & 2003257 & 8.65 & 4.7969 \\
\hline & 3.50 & 0.139986 & 57.29 & 2058950 & 11.67 & 6.4619 \\
\hline \multicolumn{2}{|c|}{ Total Err. } & & 64 & & & \\
\hline
\end{tabular}
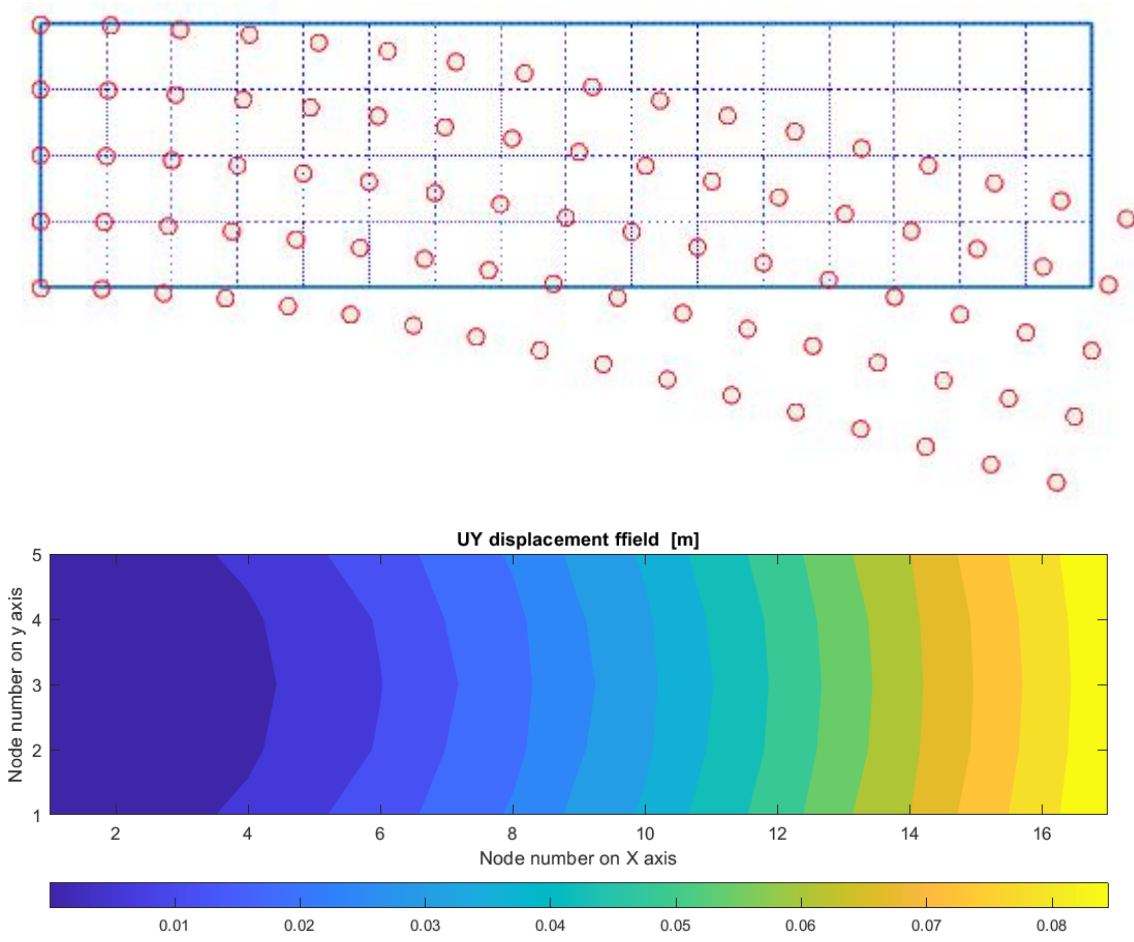

Figure 12: Deformed state and nodal displacement field, for $d_{m}=2.0, d_{n n}=0.03 \mathrm{~m}$

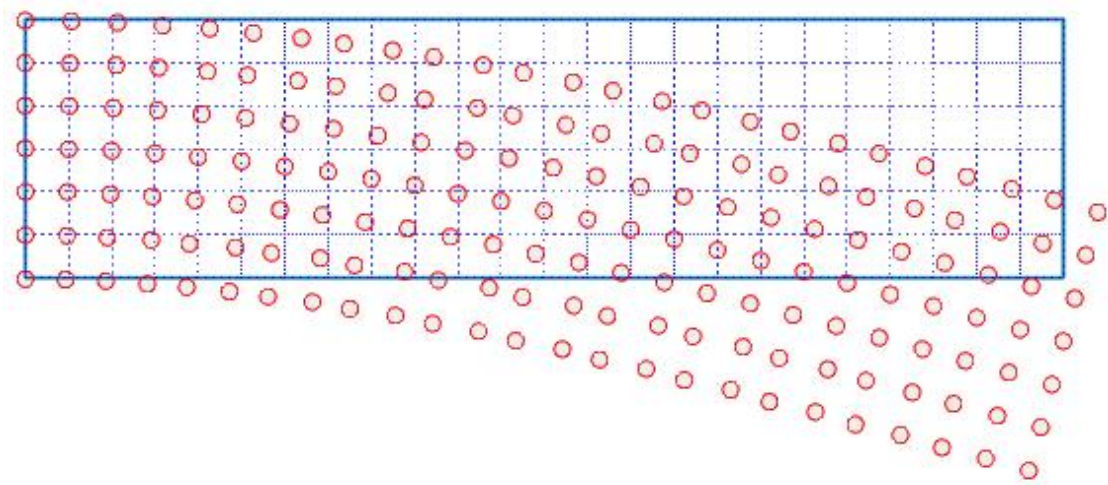

a) 


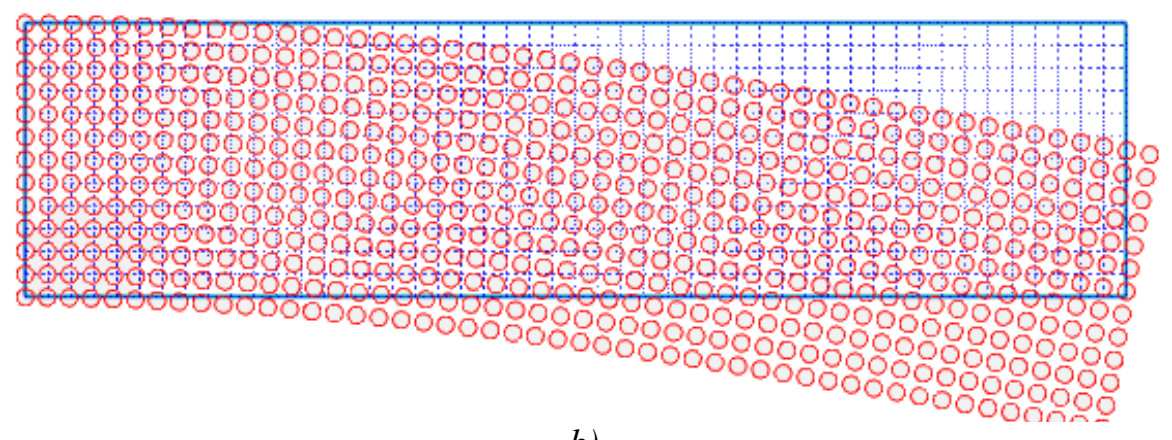

b)

Figure 13: Deformed states for $\left.\left.d_{m}=2.0, a\right) d_{n n}=0.02 \mathrm{~m}, b\right) d_{n n}=0.01 \mathrm{~m}$

The parameter $d_{m}$, which appears in the Table 1, is a scaling parameter, with a recommended value between 2 and 4, for static analysis [2], [3].

By the parameter $d_{m}$, the support domain size (constant or variable from one node to another) for the interested node $d_{m I}$ is calculated:

$$
d_{m I}=d_{\mathrm{m}} \cdot c_{I}
$$

In our researching the parameter $d_{m I}$ was constant for all nodes; also $d_{m}$, but this was taken into account by different its values. The parameter $c_{I}$ is a distance between nodes, so chosen that to have enough neighbor points as the matrix not to be singular. In the Figure 11, the deformed states and nodal displacement field are presented for the cubic spline weight function and $d_{m}=2.0$. The analysis of the results led to the following graphical representations.

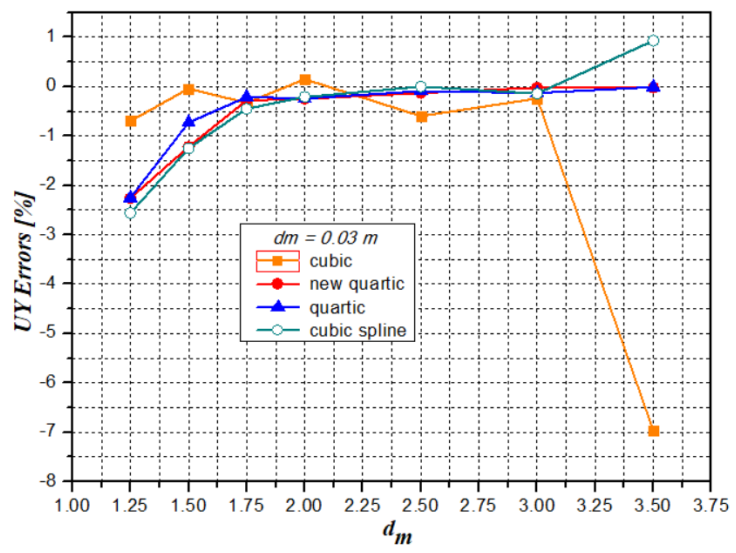

a)

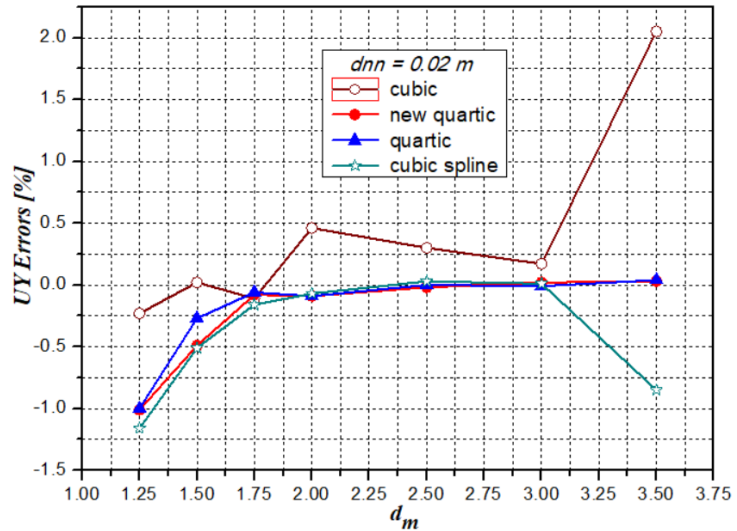

b)

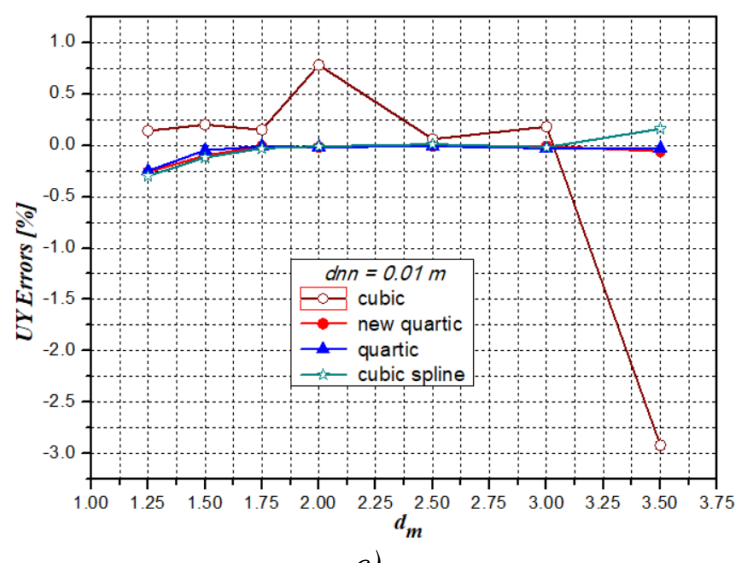

c)

Figure 14: $U Y_{\max }$ error evolution versus $d_{m}$ for some weight functions

The Figure 12 shows the deformed state for the case of cubic spline weight function and $d_{m}=2.0$.

In the Figure 13, the evolution of the $u_{y}^{\max }$ displacement errors versus support domain size (by $d_{m}$ ) is presented, for those considered internodal distances (a), b) and c)). 
We can see that the best results are obtained for the values between 1.75...3.0 for the scaling parameter $d_{m}$.

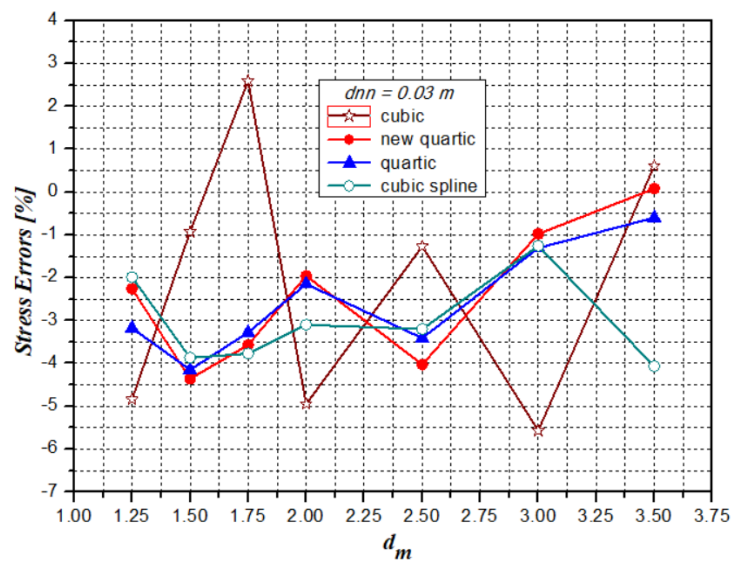

a)

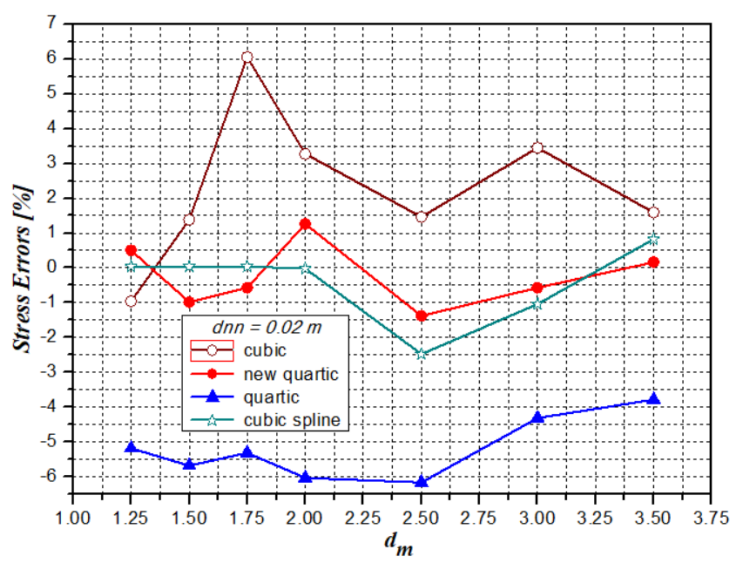

b)

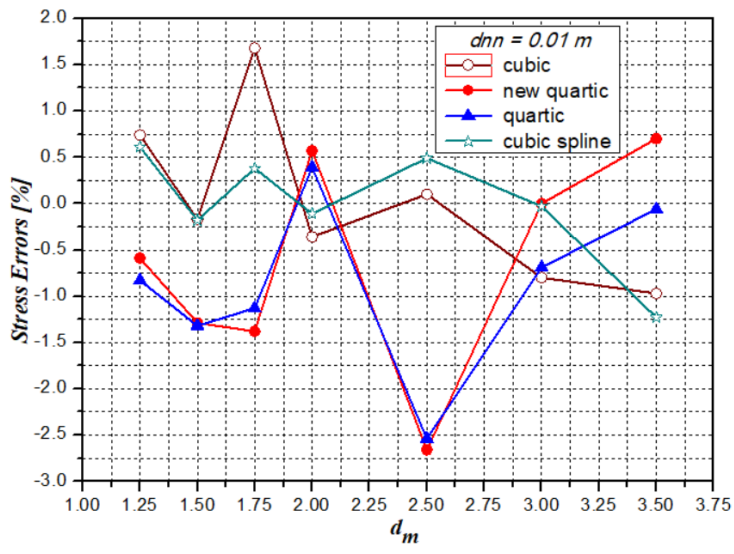

c)

Figure 15: $S X_{\max }$ error evolution versus $d_{m}$ for some weight functions and three values of internodal distance $a$ ), $b$ ) and c).

The analysis of errors, in stress calculation by EFG method shows us that these errors are greater than the calculation errors of the nodal displacements.

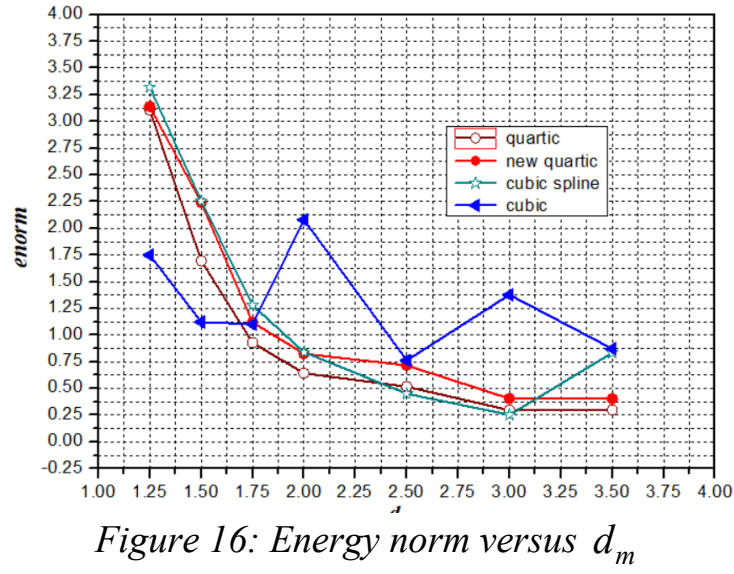

The Figure 15 shows the dependence of the energy norm error by the support domain size $\left(d_{m}\right.$ parameter $)$ for the case of internodal distance of $0.03 \mathrm{~m}$.

\section{Conclusions}

Our paper presents the most important researching results on the use of the EFG method. This numerical meshless method, being under development, being relatively new, raises in front of the user a series of aspects regarding the choice of the weight function, the choice of some of its parameters, the choice of the number of integration cells, the choice of the internodal distance and others.

The presented results allow us to conclude:

- the method is less sensitive regarding the internodal distance, if the parameters of the weight function are chosen within the limits recommended by the specialized literature;

- the calculation accuracy of the nodal displacements is superior to the calculation accuracy of the stresses;

- the internodal distance has an important role regarding the accuracy of the method results and a decisive one regarding the field of the calculated parameter values; sometimes its appearance is far from reality, even if the determined maximum values know a good precision;

- our research aimed at a greater number of weighting functions, but in this paper 
are presented only those weighting functions that have proven to be generally valid;

- Johnson's quadratic function seems very interesting, but he will have to enjoy a dedicated study on impact issues;

- Regarding the size of the support domain, our research recommends values between 1.50 to 2.50 for the $\mathrm{dm}$ parameter;

- Our study shows that out of the 4 weighted functions investigated, the cubic function should be avoided, the results of the others being better in terms of appearance and stability.

This paper can be useful to any user of the EFG method, even a beginner, if previously, the theoretical fundamentals of the method (developed more than in this paper) have been studied.

\section{References List}

[1] Belytschko, T., Lu, Y.,Y., Gu, L., Element-free Galerkin methods, Int. J. Numer. Methods Engrg. 37: 229-256, 1994.

[2] Dolbow, J., Belytschko, T., An Introduction to Programming the Meshless Element Free Galerkin Method, Archives of Computational Methods in Engineering, State of the art reviews, Vol. 5, 3, 207-241, 1998.

[3] Liu, G., R., Meshfree Methods, Moving Beyond the Finite Element Method, 2nd ed., CRC Press, New York, 2010.

[4] Liu, G., R., Liu, M., B., Smoothed Particle Hydrodynamics A Meshfree Particle Method, World Scientific Publishing C. Pte. Ltd., London, 2009.

[5] Liu, G., R., Gu, Y., T., An Introduction to Meshfree Methods and Their Programming, Springer, P.O. Box 17, 3300 AA Dordrecht, The Netherlands. 2005.

[6] Năstăsescu, V., Bârsan, Gh., Metoda particulelor libere în analiza numerică a mediilor continue, Editura AGIR, București, 2015.

[7] Năstăsescu, V., Bârsan, Gh., Metoda elementelor libere Galerkin în analiza structurilor, Editura Academiei Române, București, 2018.

[8] Pandey, S., S., Kasundra, P., K., Daxini, S., D., Introduction of Meshfree Methods and Implementation of Element Free Galerkin (EFG) Method to Beam Problem, International Journal on Theoretical and Applied Research in Mechanical Engineering (IJTARME), Volume-2, Issue-3, 2013, pg. 85-89.

[9] Saeid Zahiri, Meshfree Methods, Numerical Analysis - Theory and Application, Shanghai Jiao Tong University, September 2011, DOI: 10.5772/23509 - Source: InTech

[10] Singh, I. V., Meshless EFG Method in Three-Dimensional Heat Transfer Problems: A numerical Comparison, Cost and Error Analysis, Numerical Heat Transfer, Part A, 46: 199-220, 2004, Copyright (CTaylor \& Francis Inc., ISSN: 1040-7782 print=1521-0634 online, DOI: 10.1080/10407780490457437

[11] Shaofan Li, Wing Kam Liu, Meshfree Particle Methods, Springer-Verlag Berlin Heidelberg 2004, 2007.

[12] Timoshenko, S., P., Goodier, J., N., Theory of Elasticity (Third edition), New York: McGraw Hill, 1970.

[13] Voinea, R., Voiculescu, D., Ceaușu, V., Elasticitate și plasticitate, Editura Printech, București, 2000 .

[14] Wang, J. G., Liu, G., R., On the optimal shape parameters of radial basis functions used for 2-D meshless methods, Computer Methods in Applied Mechanics Engineering. 191 (2002) 2611-2630, pg. 2611-2630, www.elsevier.com/locate/cma 